\title{
Plug and Play Distributed Model Predictive Control Based on Distributed Invariance and Optimization
}

\author{
M.N. Zeilinger ${ }^{1}$, Y. Pu $^{2}$, S. Riverso ${ }^{3}$, G. Ferrari-Trecate ${ }^{3}$, C.N. Jones ${ }^{2}$
}

\begin{abstract}
This paper presents a method for plug-and-play distributed MPC of a network of interacting linear systems. The previously introduced idea of plug and play control addresses the challenge of performing network changes in the form of subsystems that are joining or leaving the network during closed-loop operation, while maintaining stability and constraint satisfaction. This work extends these ideas to an iterative distributed MPC scheme for systems with strong coupling by employing a recently proposed method for distributed MPC that takes the coupling dynamics into account in the form of time-varying terminal sets and distributed optimization. A distributed synthesis procedure for the update of the local control laws is proposed together with a transition scheme preparing the system for the upcoming modifications. This enables automatic plug-and-play operation, including rejection if the new network topology is infeasible. Both the synthesis and online control are entirely distributed and are only based on local information on the subsystems and their coupled neighbors. Finally, the proposed scheme is applied to the problem of frequency control in a power network.
\end{abstract}

\section{INTRODUCTION}

Distributed control is a promising tool to overcome the limiting computational complexity and communication requirements associated with centralized control of large-scale networked systems. This paper presents an approach for distributed linear Model Predictive Control (MPC) with plug and play capabilities, enabling network changes by subsystems joining or leaving the network, while ensuring stability and constraint satisfaction of the global system. The proposed scheme makes plug and play MPC available for networks with strongly coupled subsystems by means of distributed invariance and optimization and allows for an entirely distributed controller synthesis.

The control of large complex networks represents one of the key challenges in order to cope with many relevant control problems, examples include electric power, water resources, gas and fuel distribution, transportation or telecommunication networks. In this context, Model Predictive Control offers an attractive tool, being a high performance control scheme for constrained systems that allows

The research of M.N. Zeilinger has received funding from the EU FP7 under grant agreement no. PIOF-GA-2011-301436-"COGENT" and the research of G. Ferrari-Trecate and S. Riverso from the EU FP7/2007-2013 under grant agreement no. 257462 HYCON2 Network of excellence.

${ }^{1}$ Department of Electrical Engineering and Computer Sciences, UC Berkeley, CA 94702, USA and Department of Empirical Inference, Max Planck Institute for Intelligent Systems, 72076 Tübingen, Germany melanie.zeilingerdeecs.berkeley.edu

${ }^{2}$ Laboratoire d'Automatique, École Polytechnique Fédérale de Lausanne, 1015 Lausanne, Switzerland \{y.pu, colin. jones $\}$ depfl.ch

${ }^{3}$ Dipartimento di Ingegneria Industriale e dell'Informazione, Università deli Studi di Pavia, 27100 Pavia, Italy \{stefano.riverso, giancarlo.ferrari\} dunipv.it for the formulation of a variety of control specifications and objectives. Distributed MPC has recently received significant attention and a range of methods for different network and communication systems have been proposed [1]-[4]. Adopting the scheme in [5], the techniques can be classified according to the information exchange into non-iterative and iterative algorithms and the type of objective into cooperative and non-cooperative algorithms. In this paper we consider an iterative, cooperative MPC method based on distributed optimization.

This work is motivated by the recently introduced concept of plug and play decentralized or distributed MPC [6], [7]. While the main focus of distributed MPC so far has been on the control of networks with constant topology, the concept of plug and play MPC, see also [8], considers network changes by subsystems that want to join or leave the network, while ensuring feasibility and stability of the global system. By providing an automatic and distributed redesign of the control laws in response to changing network conditions, plug and play MPC is an attractive scheme for modern control systems of increasing complexity. The methods in [6], [7] are based on the idea of treating the coupling terms as disturbances using robust MPC schemes and are therefore suited for weak coupling between subsystems.

This paper extends the ideas of distributed plug and play MPC to systems with strong coupling by employing a recently proposed scheme for distributed invariance and stability [9], see also [10], [11] for related concepts, where the coupling dynamics are taken into account by means of timevarying distributed terminal sets and distributed optimization [12]. The envisioned plug and play capabilities impose two key challenges: 1. The local control laws have to be redesigned for the modified dynamics, involving information that is available locally. 2. Feasibility of the network change has to be assessed and, if permitted, performed during online operation. While common distributed MPC techniques such as [9] could be used for controller redesign, they would require a new controller synthesis for the entire network after each modification and, most importantly, they do not cover the problem of transitioning the system to the new configuration. The proposed method addresses these challenges by means of a preparation phase ensuring recursive feasibility and stability during plug-and-play operation. In addition, a distributed synthesis method is presented, where the redesign of the local control laws is restricted to subsystems that are directly influenced by the modification, i.e. neighbors to the joining/leaving subsystems, in order to reduce the preparation time and allow for quick plug and play operation. 
After introducing the problem setup and the distributed MPC approach [9] in Section II, serving as a starting point for the proposed method, the conditions for the redesign of the local control laws and the transition phase are derived in Section III and a method for their distributed synthesis is proposed in Section IV. Section V demonstrates the performance of the method using a simulation example.

\section{Preliminaries}

Notation: We denote by $\operatorname{diag}\left(S_{1}, S_{2}, \ldots, S_{n}\right)$, a blockdiagonal matrix with matrices $S_{1}$ to $S_{n}$ on the main diagonal and zeros everywhere else. A function $f: \mathbb{R}_{+} \rightarrow \mathbb{R}_{+}$ belongs to class $\mathcal{K}$ if it is continuous, strictly increasing and if $f(0)=0$. A function $f: \mathbb{R}_{+} \rightarrow \mathbb{R}_{+}$belongs to class $\mathcal{K}_{\infty}$ if $f \in \mathcal{K}$ and if $\lim _{s \rightarrow \infty} f(s)=\infty$.

Consider a linear time-invariant system

$$
\mathbf{x}^{+}=A \mathbf{x}+B \mathbf{u}
$$

with state vector $\mathbf{x} \in \mathbb{R}^{n}$ and input vector $\mathbf{u} \in \mathbb{R}^{m}$ that consists of $\mathrm{M}$ dynamically coupled subsystems with the following dynamics

$$
x^{[i]+}=\sum_{j=1}^{M} A_{i j} x^{[j]}+B_{i} u^{[i]},
$$

where $x^{[i]} \in \mathbb{R}^{n_{i}}$ denotes the state and $u^{[i]} \in \mathbb{R}^{m_{i}}$ denotes the input of the $i$ 'th subsystem, $i \in \mathcal{M}:=\{1, \ldots, M\}$, i.e. $\mathbf{x}=\left[x^{[1]}, \ldots, x^{[M]}\right], \sum_{i=1}^{M} n_{i}=n, \mathbf{u}=\left[u^{[1]}, \ldots, u^{[M]}\right]$, $\sum_{i=1}^{M} m_{i}=m, A_{i j} \in \mathbb{R}^{n_{i} \times n_{j}}, B_{i} \in \mathbb{R}^{n_{i} \times m_{i}}, i, j \in \mathcal{M} . \mathcal{N}_{i}$ denotes the set of neighbors of subsystem $i$, including $i$ itself, defined as $\mathcal{N}_{i}:=\left\{j \in \mathcal{M} \mid A_{i j} \neq 0\right\}$. We denote by $x^{\mathcal{N}_{i}}$ the concatenated vector of states of all subsystems $j \in \mathcal{N}_{i}$. For a square matrix $A \in \mathbb{R}^{n \times n}$, the notation $A_{i} \in \mathbb{R}^{n_{i} \times n}$ is used to denote the matrix composed of the rows of $A$ corresponding to subsystem $i, A_{i j} \in \mathbb{R}^{n_{i} \times n_{j}}$ to denote the matrix composed of the rows of $A$ corresponding to subsystem $i$ and the columns corresponding to subsystem $j$.

Assumption II.1. Each subsystem (2) is subject to local state and input constraints

$$
x^{[i]} \in \mathbb{X}^{[i]}, u^{[i]} \in \mathbb{U}^{[i]} \quad \forall i=1, \ldots, M,
$$

where $\mathbb{X}^{[i]}, \mathbb{U}^{[i]}$ are convex sets containing the origin in the interior. This results in the constraints $\mathbf{x} \in \mathbb{X}:=\mathbb{X}^{[1]} \times \cdots \times$ $\mathbb{X}^{[M]}, \mathbf{u} \in \mathbb{U}:=\mathbb{U}^{[1]} \times \cdots \times \mathbb{U}^{[M]}$ on the global system (1).

Assumption II.2 (Communication). Two subsystems $i$ and $j$ can exchange their state information if either $j \in \mathcal{N}_{i}$ or $i \in \mathcal{N}_{j}$.

\section{A. Distributed Synthesis and Control}

The proposed method employs the approach introduced in [9] for the synthesis of a distributed MPC controller for system (1). The key benefit of the method is that it provides a distributed MPC control law with feasibility and stability guarantees while reducing conservatism and allowing for distributed optimization. This is enabled by means of decentralized terminal costs and time-varying terminal sets that can also be synthesized in an entirely distributed way, an aspect that is particularly important for the considered case of plug and play MPC, where the control laws have to be redesigned locally after a network modification.

The proposed MPC problem $\mathbb{P}^{\mathcal{M}}(\mathbf{x}(t), \boldsymbol{\alpha}(t))$ to be solved at time $t$, where $\boldsymbol{\alpha}(t)=\left[\alpha^{[1]}(t), \ldots, \alpha^{[M]}(t)\right]$, is given by

$$
\begin{array}{ll}
\min & \sum_{i=1}^{M} \sum_{k=0}^{N-1} l^{[i]}\left(x_{k}^{[i]}, u_{k}^{[i]}\right)+V_{f}^{[i]}\left(x_{N}^{[i]}\right) \\
\text { s.t. } & x_{0}^{[i]}=x^{[i]}(t), \\
& x_{k+1}^{[i]}=\sum_{j \in \mathcal{N}_{i}} A_{i j} x_{k}^{[j]}+B_{i} u_{k}^{[i]}, \\
& x_{k}^{[i]} \in \mathbb{X}^{[i]}, u_{k}^{[i]} \in \mathbb{U}^{[i]} \\
& x_{N}^{[i]} \in \mathcal{X}_{f}^{[i]}\left(\alpha^{[i]}(t)\right) \\
& \forall k=1, \ldots, N-1, \forall i \in \mathcal{M}
\end{array}
$$

where $V_{f}^{[i]}(\cdot)$ are local terminal cost functions, $\mathcal{X}_{f}^{[i]}\left(\alpha^{[i]}(t)\right):=\left\{x^{[i]} \in \mathbb{R}^{n_{i}} \mid V_{f}^{[i]}\left(x^{[i]}\right) \leq \alpha^{[i]}(t)\right\}$ are local terminal constraints taken as a time-varying level set of the local terminal cost functions and $l^{[i]}\left(x^{[i]}, u^{[i]}\right)$ are local stage costs satisfying the following standard assumption.

Assumption II.3. For all $i \in \mathcal{M}$, there exist functions $\beta_{1,2,3}^{[i]} \in \mathcal{K}_{\infty}$ such that

$$
\begin{aligned}
& \beta_{1}^{[i]}\left(\left\|x^{[i]}\right\|\right) \leq V_{f}^{[i]}\left(x^{[i]}\right) \leq \beta_{2}^{[i]}\left(\left\|x^{[i]}\right\|\right), \\
& \beta_{3}^{[i]}\left(\left\|x^{[i]}\right\|\right) \leq l^{[i]}\left(x^{[i]}, u^{[i]}\right) \quad \forall x^{[i]} \in \mathbb{X}^{[i]}, u^{[i]} \in \mathbb{U}^{[i]} .
\end{aligned}
$$

The cost and inequality constraints for each subsystem in $\mathbb{P}^{\mathcal{M}}(\mathbf{x}(t), \boldsymbol{\alpha}(t))$ are decoupled from the other subsystems and coupling is only introduced through the dynamics. Since the dynamics are structured according to the coupling and communication graph of the network, the MPC problem can be solved by means of distributed optimization techniques, returning the optimal input sequences $\left[\mathbf{u}_{0}^{*}, \ldots, \mathbf{u}_{N-1}^{*}\right]$. The local control law applied to subsystem $i$ is defined in a receding horizon fashion as $\kappa^{[i]}(\mathbf{x}, \boldsymbol{\alpha})=u_{0}^{[i] *}$.

The feasible set of the MPC problem $\mathbb{P}^{\mathcal{M}}(\mathbf{x}(t), \boldsymbol{\alpha}(t))$ is denoted by $\mathbb{X}^{\mathcal{M}}(\boldsymbol{\alpha}(t))$. Note that the local terminal costs and constraints result in the following terminal cost and terminal constraint on the global system state

$$
\begin{aligned}
& V_{f}(\mathbf{x}):=\sum_{i=1}^{M} V_{f}^{[i]}\left(x^{[i]}\right), \\
& \mathcal{X}_{f}(\boldsymbol{\alpha}):=\mathcal{X}_{f}^{[1]}\left(\alpha^{[1]}\right) \times \cdots \times \mathcal{X}_{f}^{[M]}\left(\alpha^{[M]}\right) .
\end{aligned}
$$

Since $\mathcal{X}_{f}(\boldsymbol{\alpha}(t))$ is time-varying, we make use of the timeinvariant outer approximation

$$
\mathcal{X}_{f}^{o}(\alpha):=\left\{\mathbf{x} \mid V_{f}(\mathbf{x}) \leq \alpha\right\} \supseteq \mathcal{X}_{f}(\boldsymbol{\alpha}(t))
$$

for $\sum_{i \in \mathcal{M}} \alpha^{[i]}(t) \leq \alpha \forall t$.

The local terminal cost functions $V_{f}^{[i]}\left(x^{[i]}\right)$ and terminal sets $X_{f}^{[i]}\left(\alpha^{[i]}\right)$ are chosen to ensure that the terminal cost provides a Lyapunov function and the terminal set is invariant 
for the global system under a terminal control law and in consequence provide recursive feasibility and closed-loop asymptotic stability of the overall system.

Theorem II.4 ( [9], [11]). If there exists a function $\gamma^{[i]}\left(x^{\mathcal{N}_{i}}\right)$, a terminal control law $\kappa_{f}^{[i]}\left(x^{\mathcal{N}_{i}}\right)$ and a constant $\alpha^{[i]}(0)$ for all subsystems $i \in \mathcal{M}$, as well as a global constant $\alpha$ such that the following conditions are satisfied:

1: $V_{f}(\mathbf{x})$ is a Lyapunov function for system (1) under

$$
\begin{gathered}
\kappa_{f}(\mathbf{x}):=\left[\kappa_{f}^{[1]}\left(x^{\mathcal{N}_{1}}\right), \ldots, \kappa_{f}^{[M]}\left(x^{\mathcal{N}_{M}}\right)\right], \text { i.e. } \\
V_{f}^{[i]}\left(x^{[i]+}\right)-V_{f}^{[i]}\left(x^{[i]}\right) \\
\leq-l^{[i]}\left(x^{[i]}, \kappa_{f}^{[i]}\left(x^{\mathcal{N}_{i}}\right)\right)+\gamma^{[i]}\left(x^{\mathcal{N}_{i}}\right) \\
\forall x^{[i]} \in \mathcal{X}_{f}^{[i]}, \forall i \in \mathcal{M}, \\
\sum_{i=1}^{M} \gamma^{[i]}\left(x^{\mathcal{N}_{i}}\right)=0,
\end{gathered}
$$

2: the state and input constraints are satisfied in $\mathcal{X}_{f}^{o}$, i.e.,

$$
\mathcal{X}_{f}^{o}(\alpha) \subseteq \mathbb{X} \cap\left\{\mathbf{x} \mid \kappa_{f}^{[i]}\left(x^{\mathcal{N}_{i}}\right) \in \mathbb{U}^{[i]} \forall i \in \mathcal{M}\right\},
$$

3: initially $\mathcal{X}_{f}(\boldsymbol{\alpha}(0)) \subseteq \mathcal{X}_{f}^{o}(\alpha)$, i.e.,

$$
\sum_{i \in \mathcal{M}} \alpha^{[i]}(0) \leq \alpha
$$

4: the MPC problem is feasible for the initial state, i.e.,

$$
\mathbf{x}(0) \in \mathbb{X}^{\mathcal{M}}(\boldsymbol{\alpha}(0))
$$

then, using the dynamics

$$
\alpha^{[i]+}=\alpha^{[i]}+\gamma^{[i]}\left(x^{\mathcal{N}_{i}}\right)
$$

for the size of the local terminal sets, the closed-loop system (1) under $\kappa(\mathbf{x}, \boldsymbol{\alpha})$ starting at the initial state $\mathbf{x}(0)$ is asymptotically stable.

Remark II.5. The initial condition in (8d) together with the dynamics in (9) ensure that $0 \leq \alpha^{[i]}(t)$ and $0 \leq$ $\sum_{i \in \mathcal{M}} \alpha^{[i]}(t) \leq \alpha$ for all $t, i \in \mathcal{M}$, i.e. $\mathcal{X}_{f}(\boldsymbol{\alpha}(t)) \subseteq \mathcal{X}_{f}^{o}(\alpha)$, and therefore provide feasibility with respect to the state and input constraints in $\mathcal{X}_{f}(\boldsymbol{\alpha}(t))$.

It was shown in [9] that terminal control laws, local terminal costs and constraints satisfying the conditions (8a)(8e) can be synthesized in a distributed fashion by means of distributed optimization.

\section{Plug AND Play MPC}

In this section we extend the previously introduced scheme for distributed synthesis and control to offer plug and play capabilities, i.e. the ability to perform network changes during closed-loop operation by one or multiple subsystems that are added or removed from system (1). It is assumed that the topology changes are not immediate, but that a desired modification is indicated to the neighbors of the subsystems plugging in or out of the network, in the following called a plug and play $(\mathrm{P} \& \mathrm{P})$ request, allowing the system to prepare for the desired system change. The goal is to perform the network modification, while maintaining stability and recursive feasibility of the global system at all times, called a P\&P operation. Achieving this goal requires not only to adapt the local control laws to the modified system, but also to ensure that the system state is feasible for the modified MPC problem when applying the new control law. This is achieved by means of two preparation steps:

1) distributed redesign of the local controllers for the (virtually) modified system

2) transition phase, where the system is controlled to a steady-state, at which the P\&P operation can be performed, i.e. the subsystems plug in or out and the new control law is applied to the modified system.

In order to allow for quick plug-and-play operation and keep delays for network preparation small, we only allow the neighboring subsystems to redesign their local controllers, all other subsystems continue with the local terminal cost and constraints computed for the current network configuration.

At the time when a $\mathrm{P} \& \mathrm{P}$ request is sent, the current system has $\mathrm{M}$ subsystems with dynamics (2), applying the local control law $\kappa^{[i]}(\mathbf{x}, \boldsymbol{\alpha})$ resulting from Problem $\mathbb{P}^{\mathcal{M}}(\mathbf{x}, \boldsymbol{\alpha})$, where the current state $\mathbf{x}(0)$, the current local terminal cost functions $V_{f}^{[i]}(\cdot)$, control laws $\kappa_{f}^{[i]}(\cdot)$ and sets $\mathcal{X}^{[i]}\left(\alpha^{[i]}\right)$ satisfy conditions (8a)-(8e).

The set of subsystems that want to plug in or out are denoted with $\mathcal{P}$, the set of neighboring subsystems not including $\mathcal{P}$ with $\mathcal{N}_{\mathcal{P}}:=\bigcup_{j \in \mathcal{P}} \mathcal{N}_{j} \backslash \mathcal{P}$. Let the set of subsystems for which local controllers have to be redesigned or newly synthesized be denoted by $\mathcal{C}$ and the set of subsystems that remain unchanged with $\mathcal{R}:=\mathcal{M} \backslash \mathcal{C}$. We distinguish two cases:

- Plug-in request: $\mathcal{C}:=\mathcal{N}_{\mathcal{P}} \cup \mathcal{P}$,

- Plug-out request: $\mathcal{C}:=\mathcal{N}_{\mathcal{P}}$.

This results in the dynamics

$$
x^{[i]+}=\sum_{j \in \mathcal{N}_{i}^{\text {mod }}} A_{i j} x^{[j]}+B^{[i]} u^{[i]}
$$

of the modified system with subsystems $\mathcal{M}^{\text {mod }}:=$ $\left\{1, \ldots, M^{\text {mod }}\right\}$, where $\mathcal{N}_{i}^{\text {mod }}$ denotes the modified set of neighbors of subsystem $i$ with $\mathcal{N}_{i}^{\text {mod }}=\mathcal{N}_{i} \forall i \in \mathcal{R}$. All quantities associated with the modified system after the addition/removal of the new subsystems are in the following indicated with $\tilde{r}$, e.g. the redesigned terminal cost is $\tilde{V}_{f}^{[i]}(\cdot)$.

Remark III.1. Although not explicitly considered in (10), the preparation scheme proposed in the following allows for the dynamics of the neighboring systems themselves, i.e. $A_{i i}, i \in \mathcal{C}$, to change with the network modification.

In the remainder of this section it is shown how the current system and control law can be modified, such that all conditions in Theorem II.4 are satisfied, in which case the plug and play operation can be permitted and feasibility and asymptotic stability of the modified system (10) under the modified local control laws is guaranteed. If the conditions cannot be satisfied the P\&P request is rejected.

The proposed procedure is summarized in Algorithm 1. 


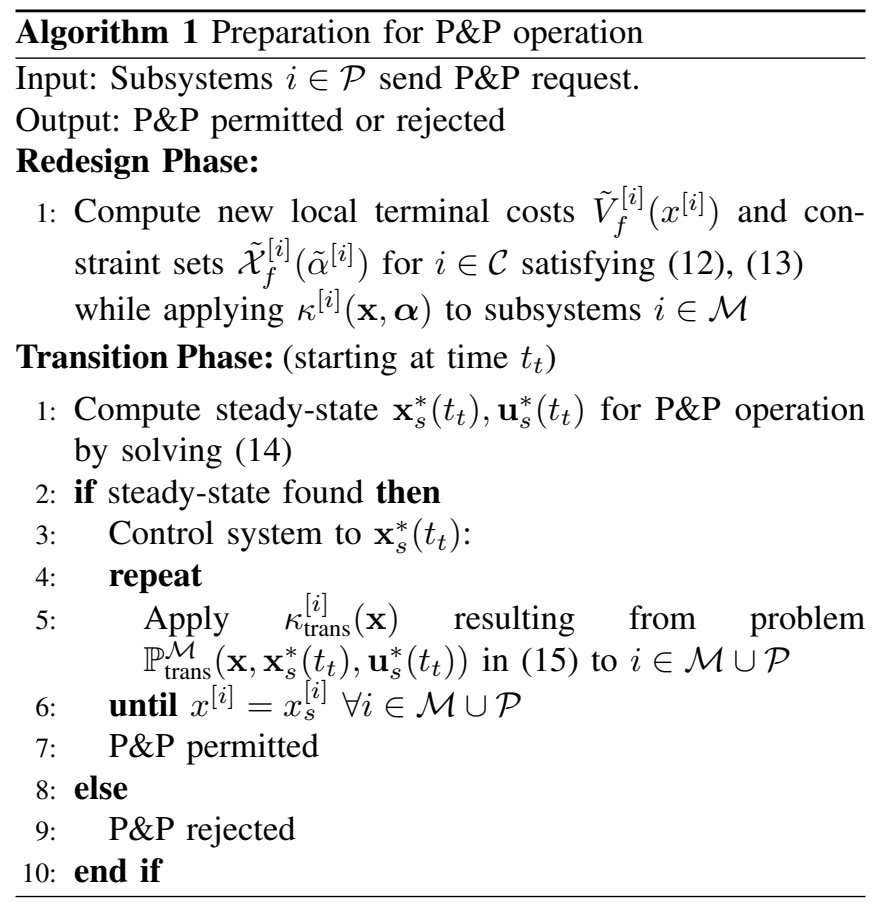

\section{A. Redesign of local controllers}

First, we derive conditions for redesigning or synthesizing local controllers for the subsystems in $\mathcal{C}$, such that conditions (8a)-(8d) are satisfied. New local terminal cost functions $\tilde{V}_{f}^{[i]}(\cdot)$ and terminal control laws $\tilde{\kappa}_{f}^{[i]}(\cdot)$ are computed for all $i \in \mathcal{C}$ such that the new global terminal cost again provides a Lyapunov function for the modified system (10) (conditions (8a)-(8b)). In order to avoid recomputation of $\alpha$, which is dependent on all subsystems, it is simultaneously imposed that the set size $\alpha$ for $\tilde{\mathcal{X}}_{f}^{o}(\alpha)$ is feasible with respect to the state and input constraints (condition (8c)), where

$$
\tilde{\mathcal{X}}_{f}^{o}(\alpha):=\left\{\mathbf{x} \mid \sum_{i \in \mathcal{M}^{\text {mod }}} \tilde{V}_{f}^{[i]}\left(x^{[i]}\right) \leq \alpha\right\} .
$$

All subsystems in $\mathcal{R}$ keep their previous setup, i.e. $\tilde{V}_{f}^{[i]}(\cdot)=$ $V_{f}^{[i]}(\cdot), \tilde{\kappa}_{f}^{[i]}(\cdot)=\kappa_{f}^{[i]}(\cdot)$ and $\tilde{\gamma}^{[i]}(\cdot)=\gamma^{[i]} \forall i \in \mathcal{R}$. Recall that the current local terminal costs satisfy $\sum_{i \in \mathcal{M}} \gamma^{[i]}\left(x^{\mathcal{N}_{i}}\right)=0$.

Lemma III.2. Consider the dynamics (10). If there exist terminal control laws $\tilde{\kappa}_{f}^{[i]}\left(x^{\mathcal{N}_{i}^{\text {mod }}}\right)$ and functions $\tilde{\gamma}^{[i]}\left(x^{\mathcal{N}_{i}^{\text {mod }}}\right), \tilde{V}_{f}^{[i]}\left(x^{[i]}\right) \forall i \in \mathcal{C}$, such that

$$
\begin{gathered}
\tilde{V}_{f}^{[i]}\left(x^{[i]+}\right)-\tilde{V}_{f}^{[i]}\left(x^{[i]}\right) \\
\leq-l^{[i]}\left(x^{[i]}, \tilde{\kappa}_{f}^{[i]}\left(x^{\mathcal{N}_{i}^{\text {mod }}}\right)\right)+\tilde{\gamma}^{[i]}\left(x^{\mathcal{N}_{i}^{\text {mod }}}\right) \\
\forall \mathbf{x} \in \tilde{\mathcal{X}}_{f}^{o}(\alpha), \forall i \in \mathcal{C}, \\
\sum_{i \in \mathcal{C}} \tilde{\gamma}^{[i]}\left(x^{\mathcal{N}_{i}^{\text {mod }}}\right)=-\sum_{i \in \mathcal{R}} \gamma^{[i]}\left(x^{\mathcal{N}_{i}}\right) \forall \mathbf{x} \in \tilde{\mathcal{X}}_{f}^{o}(\alpha), \\
\tilde{\mathcal{X}}_{f}^{o}(\alpha) \subseteq \mathbb{X} \cap\left\{\mathbf{x} \mid \tilde{\kappa}_{f}^{[i]}\left(x^{\mathcal{N}_{i}^{\text {mod }}}\right) \in \mathbb{U}^{[i]} \forall i \in \mathcal{M}^{\text {mod }}\right\},
\end{gathered}
$$

then $\tilde{V}_{f}(\mathbf{x})=\sum_{i \in \mathcal{M}^{\text {mod }}} \tilde{V}_{f}^{[i]}\left(x^{[i]}\right)$ is a Lyapunov function in $\tilde{\mathcal{X}}_{f}^{o}(\alpha)$ for the modified global system (10) with subsystems $\mathcal{M}^{\text {mod }}$ under the local terminal control laws $\tilde{\kappa}_{f}^{[i]}\left(x^{\mathcal{N}_{i}^{\text {mod }}}\right)$.
Proof: Follows directly from the definition of $\tilde{V}_{f}(\mathbf{x})$, the fact that $\tilde{V}_{f}^{[i]}(\cdot)=V_{f}^{[i]}(\cdot) \forall i \in \mathcal{R}$ satisfying (8a) and conditions (12a), (12b) using Assumption II.3 (Theorem III.4 in [11]).

As a next step we consider the redesign of the local terminal constraints such that condition (8d) is satisfied. The local terminal sets are again taken as level sets of the local terminal costs and redesign consists in choosing new set sizes $\tilde{\alpha}^{[i]}$, resulting in the local terminal sets $\tilde{\mathcal{X}}_{f}^{[i]}\left(\tilde{\alpha}^{[i]}\right):=$ $\left\{x^{[i]} \mid \tilde{V}_{f}^{[i]}\left(x^{[i]}\right) \leq \tilde{\alpha}^{[i]}\right\}$. The current state of the closed loop system under the local control laws $\kappa^{[i]}(\mathbf{x}, \boldsymbol{\alpha})$ is likely to be infeasible for the MPC problem with modified terminal constraints, independent of the set size. In order to resolve this issue, a transition steady-state will be proposed in the following, which offers the opportunity to reallocate $\alpha$ to the local sets subject to the condition $\sum_{i \in \mathcal{M}^{\text {mod }}} \tilde{\alpha}^{[i]} \leq \alpha$. For simplicity, we take

$$
\tilde{\alpha}^{[i]}=\frac{1}{M^{\mathrm{mod}}} \alpha \forall i \in \mathcal{M}^{\mathrm{mod}} .
$$

\section{B. Transition Phase}

After redesigning the local control laws, the crucial condition that remains to be satisfied is (8e). This is addressed by introducing a transition phase, where first a feasible steadystate that allows for $\mathrm{P} \& \mathrm{P}$ operation is computed and then the system is controlled to this steady-state.

1) Computation of the steady-state: The steady-state $\left(\mathbf{x}_{s}, \mathbf{u}_{s}\right)$ is chosen such that it can be reached from the current system state $\mathbf{x}$ under the previous dynamics (2) and starting from the steady-state, there exists an input sequence and corresponding state trajectory for the modified system (10) that is feasible for the constraints and the redesigned local terminal sets. These requirements are captured in the following optimization problem

$$
\begin{aligned}
\min & \sum_{i \in \mathcal{M} \cup \mathcal{P}} f^{[i]}\left(x_{s}^{[i]}, u_{s}^{[i]}, x^{[i]}\right) \\
\text { s.t. } & x_{s}^{[i]}=\sum_{j \in \mathcal{N}_{i}} A_{i j} x_{s}^{[j]}+B^{[i]} u_{s}^{[i]}, \\
& x_{k+1}^{[i]}=\sum_{j \in \mathcal{N}_{i}} A_{i j} x_{k}^{[j]}+B^{[i]} u_{k}^{[i]}, \\
& x_{0}^{[i]}=x^{[i]}, x_{N}^{[i]}=x_{s}^{[i]}, x_{k}^{[i]} \in \mathbb{X}^{[i]}, u_{k}^{[i]} \in \mathbb{U}^{[i]}, \\
& x_{N+k+1}^{[l]}=\sum_{j \in \mathcal{N}_{i}^{\bmod }} A_{l j} x_{N+k}^{[j]}+B^{[l]} u_{N+k}^{[l]}, \\
& x_{N+k}^{[l]} \in \mathbb{X}^{[l]}, u_{N+k}^{[l]} \in \mathbb{U}^{[l]}, x_{N+N}^{[l]} \in \tilde{X}_{f}^{[l]}\left(\tilde{\alpha}^{[l]}\right), \\
& k=1, \ldots, N-1, \forall i \in \mathcal{M} \cup \mathcal{P}, \forall l \in \mathcal{M}^{\bmod } .
\end{aligned}
$$

The cost functions $f^{[i]}(\cdot)$ can be chosen to realize different desired objectives, e.g.

- $f^{[i]}\left(x_{s}^{[i]}, u_{s}^{[i]}, x^{[i]}\right):=\left\|x_{s}^{[i]}\right\|_{2}^{2}$ will keep the steady-state as close as possible to the original target, which is here given by the origin, with the goal of reducing modifications to the desired system behavior.

- $f^{[i]}\left(x_{s}^{[i]}, u_{s}^{[i]}, x^{[i]}\right):=\left\|x_{s}^{[i]}-x^{[i]}\right\|_{2}^{2}$ will keep the steadystate as close as possible to the current state, with the goal of allowing for quick plug-and-play operation. 
If a steady-state permitting P\&P operation exists, problem (14) is feasible and we denote the optimal solution with $x_{s}^{[i] *}\left(t_{t}\right), u_{s}^{[i] *}\left(t_{t}\right) \forall i \in \mathcal{M}^{\text {mod }}$, where $t_{t}$ is the start time of the transition phase. Since the cost and inequality constraints in (14) are decoupled and the subsystems are only coupled by the dynamics, the problem can be solved by means of distributed optimization.

2) Regulation to the transition steady-state: The system is controlled to $\mathbf{x}_{s}^{*}\left(t_{t}\right), \mathbf{u}_{s}^{*}\left(t_{t}\right)$ by applying the local control law $\kappa_{\text {trans }}^{[i]}(\mathbf{x})$ resulting from the standard MPC problem for regulation around a non-zero steady-state $\mathbb{P}_{\text {trans }}^{\mathcal{M}}\left(\mathbf{x}(t), \mathbf{x}_{s}^{*}, \mathbf{u}_{s}^{*}\right)$ :

$$
\begin{array}{ll}
\min & \sum_{i \in \mathcal{M}} \sum_{k=0}^{N-1} l^{[i]}\left(x_{k}^{[i]}-x_{s}^{[i] *}, u_{k}^{[i]}-u_{s}^{[i] *}\right) \\
\text { s.t. } & (4 \mathrm{~b}),(4 \mathrm{c}),(4 \mathrm{~d}), \\
& x_{N}^{[i]}=x_{s}^{[i] *} .
\end{array}
$$

We use the more restrictive terminal condition (15c) in order to avoid additional computation for adapting the terminal set to the new steady-state. Since the structure remains unchanged, the modified MPC problem can again be solved by distributed optimization. Is it important to note that if a solution to problem (14) exists, the MPC problem $\mathbb{P}_{\text {trans }}^{\mathcal{M}}$ in (15) for regulation to the steady-state is always feasible.

We conclude this section by summarizing the procedure in the following main result.

Theorem III.3. Consider the closed loop system (2), (9) under $\kappa^{[i]}(\mathbf{x}, \boldsymbol{\alpha}), i \in \mathcal{M}$ and the application of Algorithm 1 after a $P \& P$ request is made by a set of subsystems $\mathcal{P}$. Let $\tilde{\kappa}^{[i]}(\mathbf{x}, \boldsymbol{\alpha})$ be the new local receding horizon control law defined by the MPC problem with redesigned terminal costs $\tilde{V}_{f}^{[i]}(\cdot)$, terminal constraints $\tilde{\mathcal{X}}_{f}^{[i]}\left(\alpha^{[i]}\right)$ and modified dynamics (10). If the plug and play request is permitted at time $t_{p}$, the modified closed-loop system (10) under $\tilde{\kappa}^{[i]}(\mathbf{x}, \boldsymbol{\alpha})$ and the set dynamics

$$
\alpha^{[i]+}=\alpha^{[i]}+\tilde{\gamma}^{[i]}\left(x^{\mathcal{N}_{i}^{\text {mod }}}\right) \forall i \in \mathcal{M}^{\text {mod }},
$$

starting at $\mathbf{x}\left(t_{p}\right)=\mathbf{x}_{s}^{*}\left(t_{t}\right), \boldsymbol{\alpha}\left(t_{p}\right)=\tilde{\boldsymbol{\alpha}}$ obtained from (13), (14) is asymptotically stable.

Proof: Feasibility of $\mathbf{x}\left(t_{p}\right), \boldsymbol{\alpha}\left(t_{p}\right)$ for the modified MPC problem follows directly from the choice of $\mathbf{x}_{s}^{*}\left(t_{t}\right), \mathbf{u}_{s}^{*}\left(t_{t}\right)$ according to (14) and stability of the transition MPC control law $\kappa_{\text {trans }}^{[i]}(\mathbf{x})$ follows from standard MPC results [13]. Finally, convergence of the modified system starting at $\mathbf{x}\left(t_{p}\right), \boldsymbol{\alpha}\left(t_{p}\right)$ follows from Lemma III.2 and Theorem II.4.

Remark III.4. Instead of choosing $\tilde{\alpha}^{[i]}$ according to (13), it would be possible to optimize over $\tilde{\alpha}^{[i]}$ in the optimization problem (14), which would couple the subproblems in a onedimensional constraint.

Remark III.5. The transition phase can be improved by allowing for $\mathrm{P} \& \mathrm{P}$ operation when the system state is in a neighborhood $\mathcal{B}$ of the steady-state, i.e. $\mathbf{x}-\mathbf{x}_{s} \in \mathcal{B}$. In order to ensure feasibility, the optimization (14) has to be made robust against this deviation using any of the common techniques for robust MPC, such as tube-based MPC [14] or constraint tightening [15], which can be synthesized and solved by distributed optimization [16].

\section{Distributed Synthesis For Plug AND Play MPC}

In this section we present a method for the distributed synthesis of the redesigned control laws for the case of quadratic cost functions, linear terminal controllers and quadratic terminal cost functions. Let $l^{[i]}\left(x^{[i]}, u^{[i]}\right)=$ $x^{[i]^{T}} Q^{[i]} x^{[i]}+u^{[i]^{T}} R^{[i]} u^{[i]}, \kappa_{f}^{[i]}\left(x^{\mathcal{N}_{i}}\right)=K_{i} \mathbf{x}, V_{f}^{[i]}\left(x^{[i]}\right)=$ $x^{[i]} P^{[i]} x^{[i]}, \gamma^{[i]}\left(x^{\mathcal{N}_{i}}\right)=\mathbf{x}^{T} \Gamma^{[i]} \mathbf{x}$, where $Q^{[i]}, R^{[i]}$ and $P^{[i]}$ are symmetric positive definite matrices, $\Gamma^{[i]}$ are symmetric matrices (potentially indefinite) and $K_{i}, \Gamma^{[i]}$, are structured according to the coupling structure in the network, i.e. for a given $i, K_{i j}, \Gamma_{i j}^{[i]} \neq 0$ if $j \in \mathcal{N}_{i}$, otherwise 0 . Note that Assumption II. 3 is automatically satisfied by this choice. We further assume that the local constraints are polytopic sets of the form $\mathbb{X}^{[i]}:=\left\{x^{[i]} \mid G_{x, j}^{[i]} x^{[i]} \leq f_{x, j}^{[i]}, j=1, \ldots, p_{x}^{[i]}\right\}$ and $\mathbb{U}^{[i]}:=\left\{x^{[i]} \mid G_{u, j}^{[i]} u^{[i]} \leq f_{u, j}^{[i]}, j=1, \ldots, p_{u}^{[i]}\right\}$.

For the initial setup with a set of subsystems $\mathcal{M}$, it was shown in [9] that local controllers and terminal costs satisfying conditions (8a)-(8e) can be computed on the local subsystems using distributed optimization. We will now show that this can be extended to plug and play operation and all computations for the redesign of the local controllers satisfying the conditions (12) can be distributed.

Let $\tilde{P}=\operatorname{diag}\left(\tilde{P}^{[1]}, \ldots, \tilde{P}^{\left[M^{\text {mod }}\right]}\right)$. For a local matrix $Q^{[i]} \in \mathbb{R}^{n_{i} \times n_{i}}$ of subsystem $i$ we denote by $\left[Q^{[i]}\right]^{\text {lift }}$ the lifting to the global system space, with $\left[Q^{[i]}\right]_{i, i}^{\text {lift }}=Q^{[i]}$ and all other elements zero. We denote with $\tilde{A}_{i}=\left[A_{i 1}, \ldots, A_{i M^{\mathrm{mod}}}\right]$ the concatenated dynamics for subsystem $i$. For subsystems $i \in \mathcal{R}, \tilde{P}^{[i]}=P^{[i]}, \tilde{K}_{i}=K_{i}$ and let $\hat{\Gamma}^{[i]}$ denote the matrix $\Gamma^{[i]}$ computed for the system with $M$ subsystems in the space of $M^{\text {mod }}$ subsystems. Redesign of the local controllers consists in computing $\tilde{P}^{[i]}, \tilde{\Gamma}^{[i]}$ and $\tilde{K}_{i}$ for $i \in \mathcal{C}$ satisfying the constraints resulting from (12):

$$
\begin{aligned}
& \left(\tilde{A}_{i}+\tilde{B}_{i} \tilde{K}_{i}\right)^{T} \tilde{P}^{[i]}\left(\tilde{A}_{i}+\tilde{B}_{i} \tilde{K}_{i}\right)-\left[\tilde{P}^{[i]}\right]^{\text {lift }} \\
& \leq-\left[Q^{[i]}\right]^{\text {lift }}-\tilde{K}_{i}^{T} R^{[i]} \tilde{K}_{i}+\tilde{\Gamma}^{[i]} \forall i \in \mathcal{C} \\
& \sum_{i \in \mathcal{C}} \tilde{\Gamma}^{[i]}=-\sum_{i \in \mathcal{R}} \hat{\Gamma}^{[i]} \\
& G_{x, j}^{[i]} \tilde{P}^{[i]^{-1}} G_{x, j}^{[i]} \leq \frac{1}{\alpha} f_{x, j}^{[i]}{ }^{2} \forall i \in \mathcal{C}, j=1, \ldots, p_{x}^{[i]} \\
& G_{u}^{[i]} \tilde{K}_{i} \tilde{P}^{-1} \tilde{K}_{i}^{T} G_{u}^{[i]}{ }^{T} \leq \frac{1}{\alpha} f_{u, j}^{[i]}{ }^{2} \forall i \in \mathcal{C}, j=1, \ldots, p_{u}^{[i]} .
\end{aligned}
$$

In the following let $Y_{i}=\tilde{K}_{i} \tilde{P}^{-1}, \tilde{S}^{[i]}=\tilde{P}^{[i]^{-1}}, \tilde{S}=\tilde{P}^{-1}$, $\tilde{G}^{[i]}=\tilde{P}^{-1} \tilde{\Gamma}^{[i]} \tilde{P}^{-1}$.

Lemma IV.1. Conditions (17a), (17c) and (17d) can be written in form of the following set of LMIs:

$$
\left[\begin{array}{cc}
\tilde{S}^{[i]} & \tilde{A}_{i} \tilde{S}+\tilde{B}_{i} Y_{i} \\
I & {\left[Q^{[i] \frac{1}{2}} \tilde{S}^{[i]}\right]^{l i f t}} \\
& {\left[R^{[i] \frac{1}{2}} \tilde{S}^{[i]}\right]^{l i f t}} \\
\left(\tilde{A}_{i} \tilde{S}+\tilde{B}_{i} Y_{i}\right)^{T}\left[Q^{[i] \frac{1}{2}} \tilde{S}^{[i]}\right]^{l i f t}\left[R^{[i] \frac{1}{2}} \tilde{S}^{[i]}\right]^{l i j t}\left[S^{[i]}\right]^{l i f t}+\tilde{G}^{[i]}
\end{array}\right] \succeq 0,
$$




$$
\begin{aligned}
& {\left[\begin{array}{cc}
\frac{1}{\alpha} f_{x, j}^{[i]}{ }^{2} & G_{x, j}^{[i]} \tilde{S}^{[i]} \\
\tilde{S}^{[i]} G_{x, j}^{[i]} T & \tilde{S}^{[i]}
\end{array}\right] \succeq 0, j=1, \ldots, p_{x}^{[i]},} \\
& {\left[\begin{array}{cc}
\frac{1}{\alpha} f_{u, j}^{[i] 2} & G_{u, j}^{[i]} Y_{i} \\
Y_{i}^{T} G_{u, j}^{[i]} T & \tilde{S}
\end{array}\right] \succeq 0, j=1, \ldots, p_{u}^{[i]} .}
\end{aligned}
$$

Proof: For (18a) see the similar result in [9] (proof of Lemma IV.2), for (18b),(18c) see e.g. [17].

Lemma IV.2. Let $\mathcal{C}_{\mathcal{C}}:=\left\{i \in \mathcal{C} \mid \mathcal{N}_{i} \cap \mathcal{N}_{j}=\emptyset \forall j \in \mathcal{R}\right\}$ denote the subsystems in $\mathcal{C}$ that are not connected to any subsystem in $\mathcal{R}$, directly or through one of the neighbors, and $\mathcal{C}_{\mathcal{R}}:=\mathcal{C} \backslash \mathcal{C}_{\mathcal{C}}$ the subsystems in $\mathcal{C}$ that have a direct or two step connection to some subsystem in $\mathcal{R}$. Similarly $\mathcal{R}_{\mathcal{C}}:=\left\{i \in \mathcal{R} \mid \exists j \in \mathcal{C}\right.$ such that $\left.\mathcal{N}_{i} \cap \mathcal{N}_{j} \neq \emptyset\right\}$. Let $\hat{\Gamma}^{[i]} \leq D^{[i]^{T}} D^{[i]} \forall i \in \mathcal{M}$, with $D^{[i]}$ structured, i.e. $D_{i j}^{[i]} \neq 0$ if $j \in \mathcal{N}_{i}$ otherwise 0 . Condition (17b) can be enforced by means of the following sufficient LMIs

$$
\begin{aligned}
& \sum_{i \in \mathcal{C}_{\mathcal{C}}} \tilde{G}^{[i]}=0, \\
& {\left[\begin{array}{cccc}
-\sum_{i \in \mathcal{C}_{\mathcal{R}}} \tilde{G}^{[i]}-2 \tilde{S}_{\mathcal{R}} C_{1} \tilde{S}_{\mathcal{C}}-\tilde{S}_{\mathcal{R}} C_{1} \tilde{S}_{\mathcal{R}} \tilde{S}_{\mathcal{C}} D^{\left[l_{1}\right]^{T}} & \cdots & \tilde{S}_{\mathcal{C}} D^{\left[\left.l_{\mid l} \mathcal{R}_{\mathcal{C}}\right|^{]^{T}}\right.} \\
D^{\left[{ }^{1} 1\right.} \tilde{S}_{\mathcal{C}} & I & & \\
\vdots & & \ddots & \\
D^{\left[l^{\mid}\left|\mathcal{R}_{\mathcal{C}}\right|\right]} \tilde{S}_{\mathcal{C}} & & & I
\end{array}\right]} \\
& \succeq 0 \forall i \in \mathcal{C}_{\mathcal{R}} \text {. }
\end{aligned}
$$

where $\mathcal{R}_{\mathcal{C}}=\left[l_{1}, \ldots, l_{\left|\mathcal{R}_{\mathcal{C}}\right|}\right], \tilde{S}_{\mathcal{C}}=\sum_{i \in \mathcal{C}}\left[\tilde{S}^{[i]}\right]^{l i f t}$ and $\tilde{S}_{\mathcal{R}}=$ $\sum_{i \in \mathcal{R}}\left[\tilde{S}^{[i]}\right]^{l i f t}, C_{1}=\sum_{i \in \mathcal{R}_{\mathcal{C}}} \hat{\Gamma}^{[i]}$ are constant matrices.

Proof: Using the structure of $\hat{\Gamma}^{[i]},(17 \mathrm{~b})$ can be split into condition (19a) and

$$
\sum_{i \in \mathcal{C}_{\mathcal{R}}} \tilde{\Gamma}^{[i]}=-\sum_{i \in \mathcal{R}_{\mathcal{C}}} \hat{\Gamma}^{[i]} \Leftrightarrow \sum_{i \in \mathcal{C}_{\mathcal{R}}} \tilde{G}^{[i]}=-\tilde{P}^{-1} \sum_{i \in \mathcal{R}_{\mathcal{C}}} \hat{\Gamma}^{[i]} \tilde{P}^{-1} .
$$

Defining $\tilde{P}^{-1}=\tilde{S}_{\mathcal{C}}+\tilde{S}_{\mathcal{R}}$ we obtain the sufficient condition

$$
\begin{aligned}
& -\sum_{i \in \mathcal{C}_{\mathcal{R}}} \tilde{G}^{[i]}-\sum_{i \in \mathcal{R}_{\mathcal{C}}} \tilde{S}_{\mathcal{C}} D^{[i]^{T}} D^{[i]} \tilde{S}_{\mathcal{C}} \\
& -\tilde{S}_{\mathcal{R}} C_{1} \tilde{S}_{\mathcal{R}}-\tilde{S}_{\mathcal{C}} C_{1} \tilde{S}_{\mathcal{R}}-\tilde{S}_{\mathcal{R}} C_{1} \tilde{S}_{\mathcal{C}} \succeq 0,
\end{aligned}
$$

which results in (19b) by using the Schur complement.

Due to the structure of the matrices, LMI (18a) can be reduced to a small dimensional LMI of size $n_{i}+3\left|\mathcal{N}_{i}\right|$ and LMI (18c) to size $1+\left|\mathcal{N}_{i}\right|$ per subsystem $i \in \mathcal{C}$. Due to the structure of $\tilde{G}^{[i]}$, two matrices $\tilde{G}^{[i]}$ and $\tilde{G}^{[j]}$ only have overlapping entries if $\mathcal{N}_{i} \cap \mathcal{N}_{j} \neq \emptyset$ and therefore LMI (19a) and (19b) are structured and can be decomposed. The problem of computing the redesigned parameters $\tilde{P}^{[i]}, \tilde{\Gamma}^{[i]}, \tilde{K}^{[i]} \forall i \in \mathcal{C}$ for the control laws of the modified system can therefore be posed as a distributed LMI.

\section{NumericAl EXAMPLE}

In this section we illustrate the proposed method by applying it to the simulation example of a power network presented in [6], [18]. We consider a network composed of four power generation areas that are interconnected through tie-lines, to which a fifth area will be connected as schematically illustrated in Figure 1. The goal is to keep the frequency at a nominal level by automatically controlling the generation

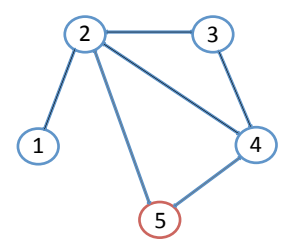

Fig. 1. Network composed of four interconnected generation areas, area five is added to the network during closed-loop operation.

when the load changes and the new generation area is connected.

The continuous-time dynamics of each generation area with primary control linearized around the equilibrium is given by [19]

$$
\dot{z}^{[i]}=\sum_{j \in \mathcal{N}_{i}} A_{i j} z^{[j]}+B_{i} v^{[i]}+L_{i} \Delta P_{L_{i}},
$$

where $z^{[i]}=\left[\Delta \phi_{i}, \Delta w_{i}, \Delta P_{m_{i}}, \Delta P_{v_{i}}\right]$ is the state, $v^{[i]}=$ $\Delta P_{r e f_{i}}$ is the control input of each generation area and $\Delta P_{L_{i}}$ is the local load change. The set of neighbors $\mathcal{N}_{i}$ of each area is given by the areas connected through tielines. The dynamic matrices and more details on the example problem can be found in [18], [19]. The local dynamics depend on the neighbors and therefore change if neighbors are added/removed, which can, however, be addressed by the proposed method (Remark III.1).

Defining the state and input as the offset from the state target $z_{r}^{[i]}=\left[0,0, \Delta P_{L_{i}}, \Delta P_{L_{i}}\right]^{T}$ and input $v_{r}^{[i]}=\Delta P_{L_{i}}$, i.e. $x^{[i]}=z^{[i]}-z_{r}^{[i]}$ and $u^{[i]}=v^{[i]}-v_{r}^{[i]}$, results in the dynamics

$$
\dot{x}^{[i]}=\sum_{j \in \mathcal{N}_{i}} A_{i j} x^{[j]}+B_{i} u^{[i]},
$$

which are then discretized with a sampling time of $1 \mathrm{sec}$ using exact discretization and treating $u^{[i]}, x^{[j]}, j \in \mathcal{N}_{i}$ as inputs. The local systems are subject to the constraints $\left\|x_{1}^{[i]}\right\|_{\infty} \leq$ $0.1 \forall i \in \mathcal{M},\left\|u^{[1]}+\Delta P_{L_{1}}\right\|_{\infty} \leq 0.5,\left\|u^{[2]}+\Delta P_{L_{2}}\right\|_{\infty} \leq$ $0.65,\left\|u^{[3]}+\Delta P_{L_{3}}\right\|_{\infty} \leq 0.65,\left\|u^{[4]}+\Delta P_{L_{4}}\right\|_{\infty} \leq 0.55$, $\left\|u^{[5]}+\Delta P_{L_{5}}\right\|_{\infty} \leq 0.5$. The weighting matrices in (4) are chosen as $Q^{[i]}=4 I, R^{[i]}=I$, where $I$ is the identity matrix of appropriate size, and the prediction horizon is $N=20$. First, matrices $P^{[i]}, \Gamma^{[i]}, K_{i}$ and constants $\alpha, \alpha^{[i]}, i \in \mathcal{M}$ defining the local control laws are computed following the method in [9] described in Section II. In the following, we apply the proposed approach for $\mathrm{P} \& \mathrm{P}$ operation, in order to add the fifth area to the network.

The local control laws for areas 2 and 4, which will be connected to area 5 , are redesigned by computing new matrices $\tilde{P}^{[i]}, \tilde{\Gamma}^{[i]}, \tilde{K}_{i}, i=2$, 4 , for the modified system dynamics and the terminal set sizes are set to $\tilde{\alpha}^{[i]}=\alpha / 5$. We consider a step change in the load for systems 1,3 and 5 at time $t=0$ of $\Delta P_{L_{1}}=-0.15, \Delta P_{L_{3}}=0.05$ and $\Delta P_{L_{5}}=-0.01$, in order to demonstrate that frequency control is achieved while changing the network topology. Following the procedure proposed in Algorithm 1, first a transition steady-state $\mathbf{x}_{s}^{*}(0), \mathbf{u}_{s}^{*}(0)$ is computed using the cost $f^{[i]}\left(x_{s}^{[i]}, u_{s}^{[i]}, x^{[i]}\right)=\left\|x_{s}^{[i]}-x^{[i]}\right\|_{2}^{2}$ in (14). A feasible steady-state is found, P\&P operation is permitted and the 

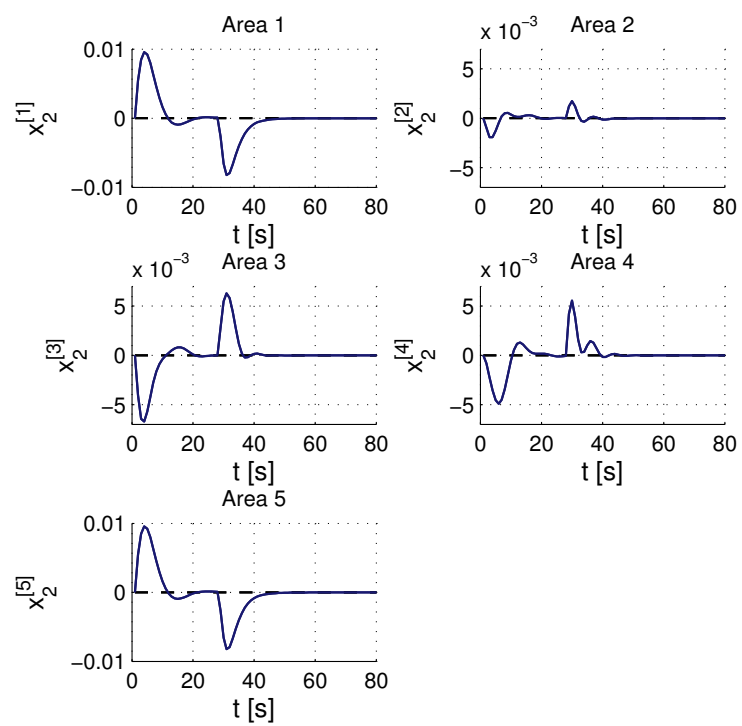

Fig. 2. Frequency deviation in all areas controlled by the proposed distributed P\&P procedure. Dashed lines indicate the transition steady-state.
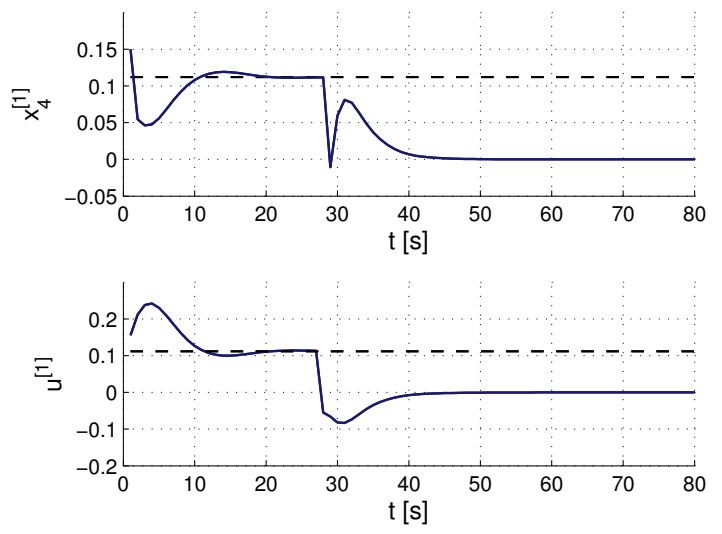

Fig. 3. Trajectory of $x_{4}^{[1]}$ of area 1 controlled by the proposed distributed $\mathrm{P} \& \mathrm{P}$ procedure, illustrating first the convergence to the transition steadystate, indicated by the dashed lines, and then to the origin.

transition phase starts at $t=0$. The system is regulated to the steady-state by applying the local control laws $\kappa_{\text {trans }}^{[i]}(\mathbf{x})$ resulting from (15). The transition phase ends at time step $t=27$, when area 5 is connected and the modified control laws $\tilde{\kappa}^{[i]}(\mathbf{x}, \boldsymbol{\alpha})$ using the redesigned local terminal costs and constraints are applied. The simulation results are shown in Figures 2-4. Figure 2 demonstrates that the main goal is achieved and the frequency deviation is successfully regulated to zero for all areas, also during the transition phase. The trajectory of the fourth state $x_{4}^{[1]}=P_{v_{1}}$ of area 1 shown in Figure 3 illustrates the convergence first to the steadystate, indicated by the dashed lines, and afterwards to the origin. The local terminal set sizes are analyzed in Figure 4, showing that starting from $\tilde{\alpha}^{[i]}$, the set sizes are dynamically changing during convergence and in particular the terminal set sizes for areas 4 and 5 are increased while those for areas 1 and 2 are decreased. The simulation example demonstrates that the distributed $\mathrm{P} \& \mathrm{P}$ method proposed in this paper allows to perform changes in the network topology during closed-loop operation, while ensuring stability at all times.

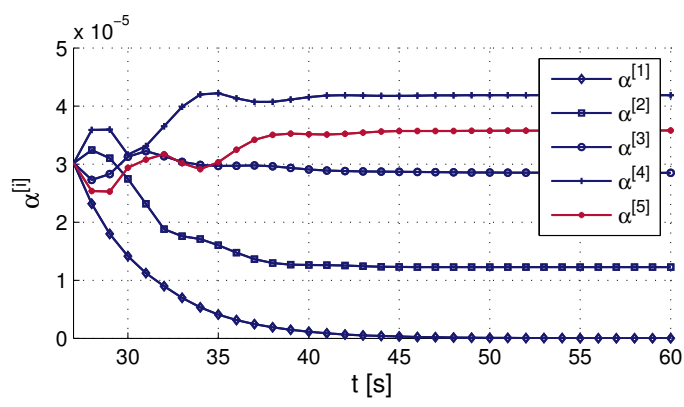

Fig. 4. Terminal set sizes $\alpha^{[i]}(t)$ for all areas, starting at $\tilde{\alpha}^{[i]}$ at $t=27$ after the transition phase, i.e. after convergence to the steady-state.

\section{REFERENCES}

[1] E. Camponogara, D. Jia, B.H. Krogh, and S. Talukdar, "Distributed Model Predictive Control," IEEE Control Systems Magazine, vol. 22, no. 1 , pp. 44-52, 2002

[2] A.N. Venkat, J.B. Rawlings, and S.J. Wright, "Distributed Model Predictive Control of Large-Scale Systems," in Assessment and Future Directions of Nonlinear Model Predictive Control. Springer, 2007, vol. 358, pp. pp 591-605.

[3] W.B. Dunbar, "Distributed Receding Horizon Control of Dynamically Coupled Nonlinear Systems," IEEE Trans. on Automatic Control, vol. 52 , no. 7 , pp. $1249-1263,2007$.

[4] M. Farina and R. Scattolini, "Distributed predictive control: A noncooperative algorithm with neighbor-to-neighbor communication for linear systems," Automatica, vol. 48, no. 6, pp. 1088-1096, 2012.

[5] R. Scattolini, "Architectures for distributed and hierarchical Model Predictive Control - A review," Journal of Process Control, vol. 19, no. 5, pp. 723-731, 2009.

[6] S. Riverso, M. Farina, and G. Ferrari-Trecate, "Plug-and-Play Decentralized Model Predictive Control for Linear Systems," IEEE Transactions on Automatic Control, p. In press, 2013.

[7] — , "Plug-and-Play Model Predictive Control based on robust control invariant sets," Università degli Studi di Pavia, Pavia, Italy, Tech. Rep., 2012. [Online]. Available: arXiv:1210.6927

[8] J. Stoustrup, "Plug \& Play Control: Control Technology towards new Challenges," in Proc. of the 10th European Control Conf., 2009, pp. $1668-1683$.

[9] C. Conte, N. Voellmy, M.N. Zeilinger, M. Morari, and C.N. Jones, "Distributed Synthesis and Control of Constrained Linear Systems," in Proc. of the American Control Conf., June 2012, pp. 6017-6022.

[10] S.V. Raković, B. Kern, and R. Findeisen, "Practical set invariance for decentralized discrete time systems," in Proc. of the 49th IEEE Conf. on Decision and Control, Atlanta, USA, 2010, pp. 3283 - 3288.

[11] A. Jokic and M. Lazar, "On decentralized stabilization of discrete-time nonlinear systems," in Proc. of the American Control Conf., 2009, pp. $5777-5782$.

[12] D.P. Bertsekas and J.N. Tsitsiklis, Parallel and Distributed Computation: Numerical Methods. Athena Scientific, 1997.

[13] D.Q. Mayne, J.B. Rawlings, C.V. Rao, and P.O.M. Scokaert, "Constrained model predictive control: Stability and optimality," Automatica, vol. 36, pp. 789-814, 2000

[14] D.Q. Mayne, M.M. Seron, and S.V. Raković, "Robust model predictive control of constrained linear systems with bounded disturbances," Automatica, vol. 41, pp. 219-234, 2005.

[15] D. Limon, T. Alamo, D.M. Raimondo, D. Muñoz de la Peña, J.M. Bravo, A. Ferramosca, and E.F. Camacho, "Input-to-State Stability: A Unifying Framework for Robust Model Predictive Control,' in Nonlinear Model Predictive Control. Springer, 2009, pp. 1-26.

[16] C. Conte, M.N. Zeilinger, M. Morari, and C.N. Jones, "Robust Distributed Model Predictive Control of Linear Systems," in Proc. of the 12th European Control Conf., July 2013, pp. 2764-2769.

[17] S. Boyd, L. El Ghaoui, E. Feron, and V. Balakrishnan, Linear Matrix Inequalities in System and Control Theory, ser. Studies in Applied Mathematics. Philadelphia, PA: SIAM, Jun. 1994, vol. 15.

[18] S. Riverso, M. Farina, and G. Ferrari-Trecate, "Plug-and-play decentralized model predictive control," Università degli Studi di Pavia, Pavia, Italy, Tech. Rep. 157/12, 2012. [Online]. Available: http://arxiv.org/abs/1302.0226

[19] H. Saadat, Power Systems Analysis, 2nd ed. New York, USA McGraw-Hill Series in Electrical and Computer Engineering, 2002. 